\title{
Analyse von Lignocellulose mittels dynamischer Differenzkalorimetrie und Infrarot - Spektrometrie
}

\author{
Helene Wulfhorst, Johannes Merseburg, Nils Tippkötter
}

Bioverfahrenstechnik, Technische Universität Kaiserslautern, Deutschland

wulfhorst@mv.uni-kl.de

\begin{abstract}
In diesem Artikel handelt es sich um die Entwicklung analytischer Methoden zur Charakterisierung der Rohstoffe für eine Bioraffinerie. Die Kalorimetrie und die Spektrometrie in Kombination mit der multivariaten Analytik wurden angewendet, um den Cellulosegehalt in den gemischten Modellproben aus Cellulose und Lignin sowie im Faserstoff nach der hydrothermalen Vorbehandlung zu analysieren. Sowohl die kalorimetrische als auch die spektrometrische Messung erlaubten den Celluloseanteil in den Modellproben zu erfassen. Die Bestimmung des Cellulosegehalts im Faserstoff war dagegen nur durch die kalorimetrische Messung möglich. Hierzu wurde eine neue Methode etabliert, die auf der Differentiellen Kalorimetrie basiert und eine schnelle Bestimmung des Cellulosegehaltes im Faserstoff ohne jegliche Probenvorbehandlung ermöglicht.
\end{abstract}

Key words: Cellulose, Kalorimetrie, Spektrometrie, Multivariate Datenanalyse

\section{Einleitung}

Holz ist ein vielversprechender nachwachsender Rohstoff für die Herstellung von Biokraftstoffen und Chemikalien. Aufgrund seiner komplexen Struktur ist jedoch eine effiziente Vorbehandlung erforderlich, um seine Hauptkomponenten in das gewünschte Endprodukt umzuwandeln. Die Erschließung von Holz ist auf unterschiedlichen Wegen möglich: Der Aufschluss kann mechanisch, thermomechanisch, chemisch oder chemischthermomechanisch erfolgen. Die mechanische Vorbehandlung wird in erster Linie durchgeführt, um das Holz zu zerkleinern. Eine Kombination der mechanischen Verfahren mit den weiteren physikalischen Methoden wie Dampfexpansion [1] und Liquid Hot Water Verfahren (LHW) ermöglicht neben der Reduktion der Partikelgröße, die Holzfaser nahezu vollständig zu desintegrieren [2].

Während der hydrothermalen Behandlung werden die Pflanzenmaterialien in einem Lösungsmittel suspendiert und bei hohen Temperaturen unter Druck erhitzt. Das LHWVerfahren verwendet dabei Wasser als Lösungsmittel. Als weitere hydrothermale Methode zum Aufschluss von Lignocellulose wird beim Organosolv-Prozess $[3,4]$ ein organisches oder wässrig/organisches Lösungsmittelgemisch mit einem Säurekatalysator $\left(\mathrm{HCl}\right.$ oder $\left.\mathrm{H}_{2} \mathrm{SO}_{4}\right)$ kombiniert. Als Lösungsmittel können Aceton, Ethylenglycol oder Tetrahydrofurfurylalkohol eingesetzt werden. Eine Weiterentwicklung des Organosolv-Verfahrens ist der Organosolv-
Ethanol-Prozess. Mit diesem Prozess, von der kanadischen Zellstoff- und Papierindustrie als Alcell囚-Aufschlussverfahren bezeichnet, wird industriell Hartholz fraktioniert. Die Weiterentwicklung verbessert die enzymatische Abbauarbeit der Kohlenhydrate und führt zu einer erheblich höheren Ausbeute an hoch qualitativem Lignin $[5,6,7]$.

Die Zusammensetzung der Faserstoffe nach hydrothermaler Vorbehandlung variiert erheblich, je nach Ausgangsmaterial und Vorbehandlungsbedingungen und beeinflusst die nachfolgenden Prozessschritte. Aus diesem Grund sind geeignete analytische Methoden erforderlich, um die Zusammensetzung verschiedener Rohstoffe für die optimale Prozessentwicklung schnell zu charakterisieren. Die herkömmlichen Methoden sind jedoch ausgesprochen zeit- und arbeitsintensiv, so dass ein hoher Bedarf an neuen Methoden besteht, die eine unkomplizierte und schnelle Rohstoffe-Charakterisierung erlauben.

So wurden in diesem Beitrag die Spektrometrie und die Kalorimetrie in Kombination mit der multivariaten Analytik zur Bestimmung des Cellulosegehalts in den Modellproben und dem Buchenholz nach hydrothermaler Vorbehandlung etabliert.

\section{Experimenteller Teil}

Die Modellproben wurden durch Mischen der definierten Mengen an reiner Cellulose (Cellulose fibrous, long, Batch\#047KO142, Sigma-Aldrich, MO, USA) und Lignin 
(Organosolv lignin, Batch\#08603PE, SigmaAldrich, MO, USA) hergestellt. Die nativen Proben bzw. die Faserstoffe wurden aus Buchenholz durch die hydrothermale Vorbehandlung nach LHW- oder OrganosolvEthanol-Verfahren sowie durch die sogenannte sequentielle Methode, welche diese beiden Verfahren kombiniert hergestellt. Zusätzlich wurden Prozessbedingungen wie die Temperatur und die Prozessdauer, sowie die Konzentration des Katalysators variiert. Die hydrothermale Vorbehandlung erfolgte in einem Hochdruckreaktor (Parr Instrument $\mathrm{GmbH}$, Germany). Bei der Vorbehandlung nach LHW Verfahren wurden 1:4 (w/w) Buchenholzpartikel in Wasser suspendiert, während die Vorbehandlung nach Organosolv-EthanolVerfahren in einer wässrigen Ethanollösung $(50 \% / 50 \%)$ erfolgte. Die Vorbehandlungstemperatur wurde zwischen $160^{\circ} \mathrm{C}-180^{\circ} \mathrm{C}$ und die -dauer zwischen $15 \mathrm{~min}-90$ min variiert. Zusätzlich wurden $2,5 \% \quad \mathrm{H}_{3} \mathrm{PO}_{4}$ bei einem der Aufschluss-Versuche nach LHW-Verfahren und $0,5 \% \quad \mathrm{H}_{3} \mathrm{PO}_{4}$ bei einem der AufschlussVersuche nach Organosolv- Ethanol Verfahren hinzugegeben.

Die Zusammensetzung der resultierenden Faserstoffe wurde durch eine Referenzmethode nach einem Standardprotokoll [8] analysiert. Bei dieser Methode, wurde die Biomasse durch die Säure hydrolysiert und anschließend die Hydrolyseprodukte darunter hauptsächlich Glucose und Xylose mittels der HPLC-Technik detektiert. Dabei wurde das Polymer Cellulose in ihre monomeren Einheiten nämlich Glucose gespalten. Folglich repräsentierte der gemessene Glucosegehalt der Proben ihren Celluloseanteil.

Die MIR-Spektren der gemischten Modellproben und der Faserstoffe wurden unter Verwendung eines FT-ATR-MIR-Spektrometers "Spektrum 100" (Perkin Elmer) über einen Wellenlängenbereich von $4000 \mathrm{~cm}^{-1}$ bis 650 $\mathrm{cm}^{-1}$ gemessen. Vor jeder Messung wurde das Spektrum von Luft als Referenz erfasst und die Untergrundabsorption von den Spektren der Proben subtrahiert. Jede Probe wurde in Dreifachbestimmung gemessen.

Für die DSC-Analyse wurde das Messgerät DSC 1 (Mettler Toledo, Gießen, Deutschland) verwendet. Dieses Gerät verfügt über einen homogen aufheizbaren bzw. abkühlbaren Ofenraum, in dem sich zwei Probenhalter befinden, die mit hochempfindlichen Temperatursensoren ausgestattet sind. Auf die Probenhalter wird jeweils ein Tiegel mit einem Durchmesser von $5 \mathrm{~mm}$ gelegt. Der erste Tiegel enthält eine Probe, der zweite Tiegel bleibt leer und dient als Referenz. Der Ofen wird permanent mit Schutzgas Stickstoff gespült. Der erzeugte Wärmestrom im Referenztiegel wird von den Temperatursensoren innerhalb der DSC erfasst. Die leere Referenzmessung dient zur Detektion des Tiegeleinflusses auf den Wärmestrom. Das Probenmaterial nimmt die Wärme auf oder ab. Dadurch ergibt sich eine Differenz zwischen der in der DSC erzeugten und der vom Sensor erfassten Temperatur. Diese Differenz wird gemessen.

Vor jeder DSC-Messung wurden die Proben eingewogen und in einem StandardAluminiumtiegel verschlossen. Anschließend wurde der Tiegel versiegelt und eine Öffnung in der Mitte des Deckels durchstochen. Das Trockengewicht der Probe beträgt ca. $5 \mathrm{mg}$. Anschließend wurden die Tiegel bei einer definierten Heizrate entsprechend dem Temperaturprofil aus Tabelle 1 erhitzt. Die chemometrische Analyse wurde mit der Software Unscrambler ${ }^{\circledR}(10.2$, CAMO Software AS, Trondheim, Norwegen) durchgeführt.

\section{Analyse der Modellproben}

Um eine geeignete Methode zur Rohstoffanalyse zu entwickeln, wurden zunächst die Modellsubstrate mit variablen Anteilen an Cellulose und Lignin mit Hilfe der Infrarot - Spektrometrie und der dynamischen Differenzkalorimetrie (DSC) untersucht.

Die DSC maß die Wärmestromdifferenzen und zeichnete diese über der Zeit auf. Die Differenz der Wärmeaufnahme bzw. -abgabe des Probenmaterials wurde über die Temperatursensoren im Innern des Gerätes erfasst. Die zeitabhängigen Temperaturgradienten wurden als exotherme oder endotherme Kurven, Peaks oder Stufen in Form von WärmestromDifferenz-Kurven dargestellt. Der exotherme Effekt beschrieb die Reaktion des Materials auf die zugeführte Wärme als eine Energieabgabe. Der endotherme Effekt beschrieb den Vorgang der Wärmeaufnahme.

Alle DSC-Kurven der Modellsubstrate zeigten einen typischen Verlauf (s. Abbildung 1 links). Der erste endotherme Peak bei einer niedrigen Temperatur von $\approx 100^{\circ} \mathrm{C}$ entsprach dem Verdampfen des Restwassers. Der nächste endotherme Peak war aufgrund von Schmelzen und Depolymerisation von Cellulose bei etwa $352{ }^{\circ} \mathrm{C}$ sichtbar. Der exotherme Effekt bei höheren Temperaturen ab ca. $400{ }^{\circ} \mathrm{C}$ wurde auf den Lignin-Anteil im Faserstoff zurückgeführt, denn er kam nur vor, wenn die Proben das Lignin enthielten, und er anstieg mit dem zunehmenden Ligningehalt. 
Tab. 1: Temperaturprofil der DSC-Analyse

\begin{tabular}{|c|c|c|c|}
\hline Segment & $\begin{array}{c}\mathrm{T}_{\text {start }}-\mathrm{T}_{\text {end }} \\
{ }^{\circ} \mathrm{C}\end{array}$ & $\begin{array}{c}\text { Heizrate } \\
\mathrm{K} / \mathrm{min}\end{array}$ & $\begin{array}{c}\text { Dauer } \\
\text { isotherm } \\
\text { min }\end{array}$ \\
\hline dynamisch & $25-40$ & 10 & - \\
\hline isotherm & 40 & - & 60 \\
\hline dynamisch & $40-25$ & 10 & - \\
\hline dynamisch & $25-600$ & 5 & - \\
\hline
\end{tabular}

Um die Enthalpie der spezifischen endo- oder exothermen Effekte zu bestimmen, wurde die Peakfläche integriert. Die Tabelle in Abbildung 1 zeigt, dass die Ligninzugabe und die entsprechende Verringerung des Cellulosegehalts pro Gramm Probe zu einer niedrigeren Enthalpie im Temperaturbereich zwischen $300{ }^{\circ} \mathrm{C}$ und $400{ }^{\circ} \mathrm{C}$ im Vergleich zur reinen Cellulose führte. Zusätzlich verschob sich das Peakmaximum hin zu den höheren Werten. Allerdings korrelierte die Enthalpie des endothermen Peaks zwischen $300{ }^{\circ} \mathrm{C}$ und $400{ }^{\circ} \mathrm{C}$ mit dem Cellulosegehalt der gemischten Proben. Darüber hinaus repräsentierten die niedrigen Standardabweichungen im Bereich von 0,16 -1 J/g eine hohe Genauigkeit der DSC-Messung. Schlussfolgernd, die Korrelation der DSC-Messergebnisse mit dem Cellulosegehalt der Modellproben kann durch die konventionelle Auswertung basierend auf der Integration der entsprechenden Peakfläche und der einfachen univariaten Korrelation erfolgen. Allerdings werden dabei die Temperaturverschiebung des Cellulosepeaks und die mögliche Überlappung der Wärmestrome von Cellulose und Lignin in den gemischten Proben möglicherweise nicht optimal erfasst.

In Abbildung 1 rechts sind die Ergebnisse der MIR-Analyse dieser Modellproben dargestellt. Um die Verschiebung der Basislinie, welche durch die Streueffekte aufgrund der unterschiedlichen Beschaffenheit der Proben zustande kommt, zu korrigieren, wurde eine SNV-Transformation der Spektren durchgeführt. Da die SNV-Vorbehandlung nur bei einer linearen Abhängigkeit der Konzentrationen angewendet werden kann, wurden die gemessenen Transmissionen im MIR-Bereich erst in die Absorption umgerechnet. Bei diesem Verfahren blieb die Form der Spektren jedoch unverändert.

Die unterschiedliche Zusammensetzung der Modellproben spiegelte sich in ihren Spektren wider. Vor allem unterschieden sich die spektralen Verläufe von Cellulose und Lignin deutlich. Man konnte die Absorptionsunterschiede besonders bei der $1210 \mathrm{~nm}$ Bande oder im Bereich zwischen 1000-1054 nm und $1400-1800 \mathrm{~nm}$ aber auch an einigen anderen Stellen im Spektrum (Abb.1B) erkennen. Bei den gemischten Proben überlappten sich jedoch die Spektren dieser Komponenten, so dass eine univariate Korrelation der Absorption mit dem Cellulosegehalt nicht möglich war. Eine multivariate Auswertung war daher nötig, um die Vorhersage der Celluloseanteile aus den Spektren der gemischten Modellproben zu ermöglichen.
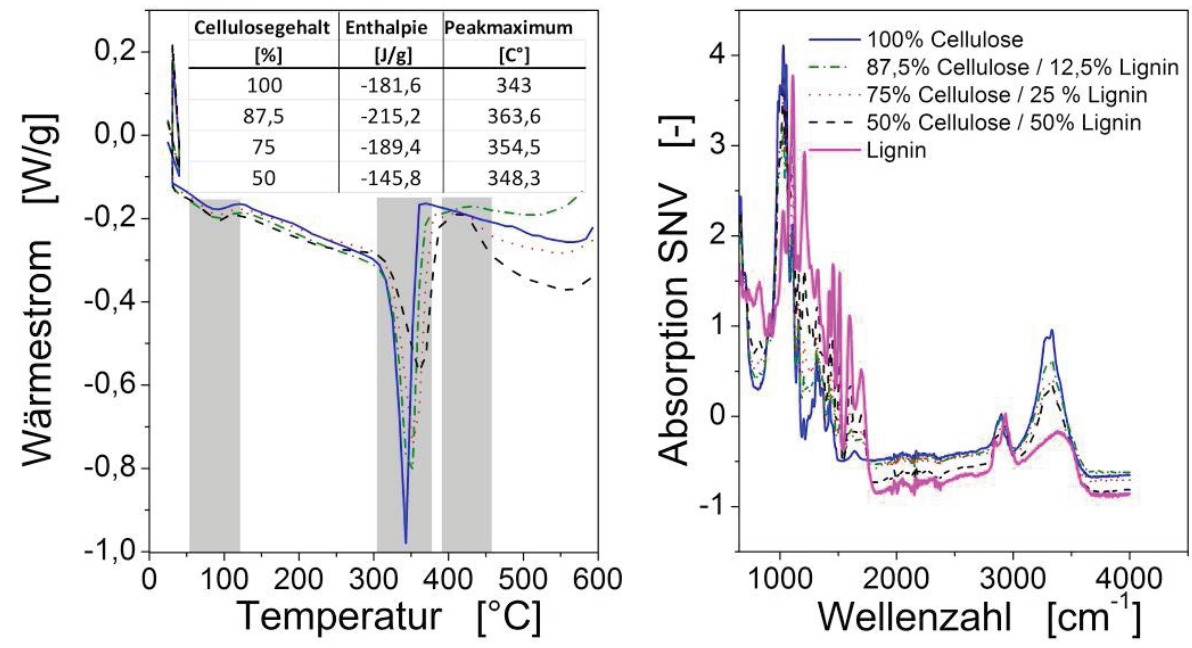

Fig. 2. Analyse der Modellsubstrate aus Cellulose und Organosolv-Lignin. Links: Wärmestromkurven, Temperaturprofil s. Tabelle 1, unter Stickstoff. Rechts: Spektren der Modellsubstrate $650 \mathrm{~cm}^{-1}-4000 \mathrm{~cm}^{-1}$. 
Aus diesem Grund wurden die Absorptionen mit den Referenzwerten bzw. dem Cellulosegehalt der jeweiligen Proben durch die PLSRegression (Partial Least Square Regression) korreliert.

Abbildung 2 zeigt das Ergebnis der PLS Regression. Zusätzlich sind darin die berechneten Regressionskoeffizienten $\left(b_{i}\right)$ für jede Wellenlänge des Spektrums als Linie dargestellt. Sie beschreiben den funktionellen Zusammenhang zwischen den $\mathrm{X}$ - und $\mathrm{Y}$ Werten. Deutlich ist zu erkennen, dass die positiven Regressionskoeffizienten dem Cellulose-Spektrum zugeordnet werden können. Diese Werte können damit den wesentlichen Beitrag zur Cellulosebestimmung liefern. Die negativen Werte entsprechen dagegen weitgehend dem Spektrum von Organosolv - Lignin. Die Absorption bei diesen Wellenlängen vermindert daher den Cellulosegehalt. Zur Vorhersage des Cellulosegehalts in den unbekannten Proben können diese Regressionskoeffizienten mit den zugehörigen Absorptionen bei jeweiligen Wellenlängen multipliziert werden.

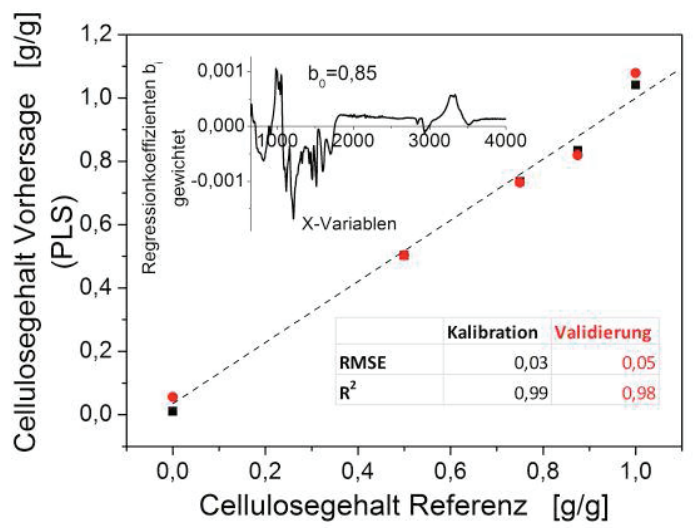

Fig. 2. Ergebnis der PLS - Regression mit den MIR-Absorptionen als Eingangsgrößen und den Messwerten der Referenzanalyse als Y-Werte.

Alle berechneten Produkte werden anschließend addiert. Die Gesamtsumme aller Regressionskoeffizienten und dem $\mathrm{b}_{0}$-Wert ergibt dann den Vorhersagewert. Deutlich zu erkennen ist, dass die Hauptinformation für den Cellulosegehalt im Wellenlängenbereich um $900 \mathrm{~nm}$ bis $1800 \mathrm{~nm}$ liegt. Der Bereich von $1800 \mathrm{~nm}$ bis $2800 \mathrm{~nm}$ kann dagegen bei der Kalibration vernachlässigt werden. Niedrige Root-Mean-Square-Error-Werte wurden sowohl für die Kalibrierung (RMSE) als auch für die Validierung berechnet. Zusammen mit den hohen $\mathrm{R}^{2}$ - Werten verdeutlichen sie, dass auch die MIR - Spektrometrie dazu geeignet ist, die Unterschiede im Cellulosegehalt der Modellproben zu erfassen und zu quantifizieren.

\section{Analyse der Faserstoffe}

Um diese Ergebnisse auf das native Substrat zu übertragen, wurde im nächsten Schritt der native Rohstoff, Buche, sequentiell durch die Kombination des LHW- und OrganosolvEthanol-Verfahren bei variablen Einstellungen vorbehandelt und mit Hilfe der DSC- und der MIR-Technik analog zu Modellsubstraten untersucht. Die Ergebnisse der konventionellen Analyse durch das herkömmliche Verfahren nach einem Standardprotokoll von Sluiter und Sluiter [8] zeigten, dass die Faserstoffe sich in ihrem Glucosegehalt deutlich unterschieden.

Die Ergebnisse der kalorimetrischen und der spektrometrischen Analyse sind in Abb. 3 zusammengefasst. Die Spektren der nativen Proben waren sehr verrauscht. Daher wurde eine Datenvorverarbeitung durch die SNV Transformation und die Savitzky - Golay Glättung durchgeführt. Die so vorbehandelten Spektren wurden anschließend analog zu Modellproben durch den PLS - Algorithmus mit den Referenzwerten korreliert. Die Auswertung der Spektren mit Hilfe des PLS - Algorithmus ergab jedoch keine zufriedenstellenden Ergebnisse. Offensichtlich war die Varianz im Glucosegehalt der nativen Proben für die spektrometrische Bestimmung nicht ausreichend.

Im Wärmestromverlauf dieser Proben waren dagegen deutliche Unterschiede zu sehen. Die linke Abbildung 3 zeigt die Thermogramme des vorbehandelten Holzes zwischen $40^{\circ} \mathrm{C}$ und $600^{\circ} \mathrm{C}$. Die Wärmeströme wurden durch das sogenannte "detrending" Verfahren vorbehandelt, um die temperaturabhängige Streuung bzw. Verschiebung zu korrigieren. Bei diesem Verfahren wurde ein quadratisches Polynom in jede Kurve gefittet, dass dann diesem Wärmestrom abgezogen wurde [9]. Alle Proben zeigten einen typischen Verlauf mit endo- und exothermen Effekten (s. Abb. 3), welche durch das Verdampfen des Restwassers aus den Poren sowie aufgrund der Degradation der Cellulose- (1), Hemicellulose- (2) und Lignin- (3) Anteile im Faserstoff vorkamen. Aufgrund der sehr heterogenen Faserstruktur und Morphologie war es jedoch schwierig die Vorgänge beim Erhitzen dieser komplexen Proben exakt zuzuordnen und zu verstehen. Die Wärmeprofile unterschiedlicher Komponenten könnten sich überlappen. Eine univariate Auswertung der DSC-Kurven würde in diesem Fall zur Fehlinterpretation der Ergebnisse führen. Aus diesem Grund wurden die Wärmeprofile multivariat mit Hilfe des PLSAlgorithmus ausgewertet und mit den Referenzwerten korreliert. Mit Hilfe der PLS wurde ein Regressionsmodell erstellt, das aus 

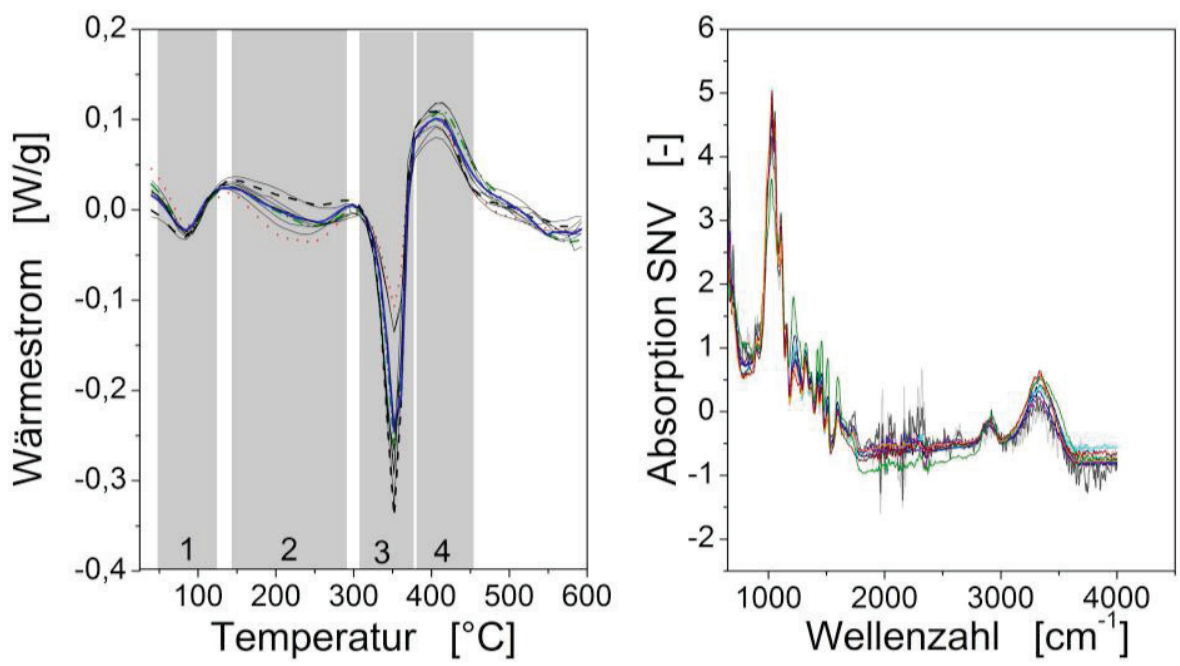

Fig. 2. Analyse von 13 verschiedenen Faserstoff - Proben. Links: Wärmestromkurven im Temperaturbereich $39^{\circ} \mathrm{C}-593^{\circ} \mathrm{C}$, unter Stickstoff. Rechts: Spektren gemessen im MIR-Bereich $650 \mathrm{~cm}^{-1}-1000 \mathrm{~cm}^{-1}$.

den DSC Messungen der Faserstoffe ihren Glucosegehalt bestimmt. Dieses Kalibrationsmodell ist in Abb. 4 dargestellt. Um das Kalibrationsmodell aufzustellen, wurden 13 Wärmestromkurven verwendet. Zwei davon wurden jedoch aufgrund ihrer sehr unterschiedlichen Profile und der hohen Restvarianz als Ausreißer identifiziert und aus der Kalibration ausgeschlossen. Diesmal waren drei Hauptkomponenten nötig, um 98,4\% der Gesamtvarianz der DSC-Daten zu erklären. Die gewichteten Regressionskoeffizienten in Abb. 4 zeigen, dass neben dem Bereich um den Schmelzpeak der Cellulose bei ca. $352^{\circ} \mathrm{C}$ weitere Bereiche für die Vorhersage des Cellulosegehalts im Faserstoff wichtig sind. Der Regressionskoeffizient $b_{0}$ wurde zu $0,305 \mathrm{~g} / \mathrm{g}$ berechnet. Das Modell zeigte einen hohen $\mathrm{R}^{2}$ Wert von 0,98 und die niedrige RSME - Fehler

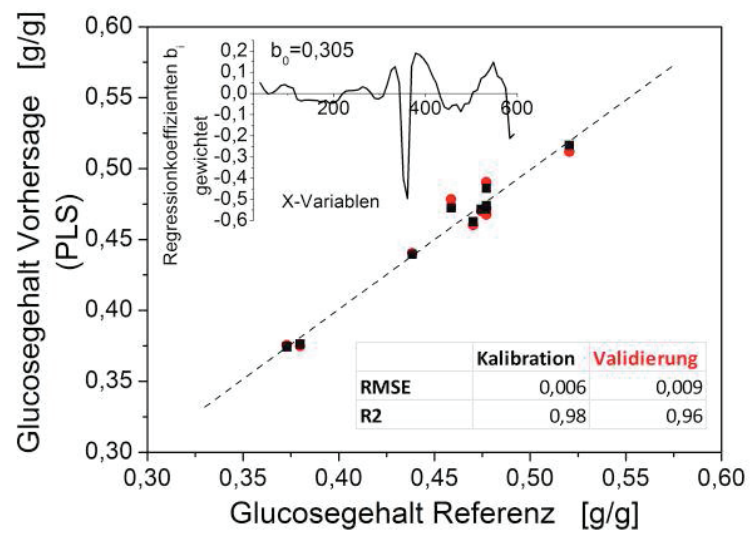

Fig. 4. Ergebnisse der PLS-Kalibration für die Kalibrierung des Glucosegehalts aus Wärmestromkurven, vorhergesagter gegen gemessenen Cellulosegehalt, berechnet aus 3 Hauptkomponenten. für die Kalibration als auch für die Validierung (s. Abb. 4).

Schlussfolgernd, das entwickelte Kalibrationsmodell erlaubte eine quantitative Vorhersage des Glucosegehaltes im hydrothermal vorbehandelten Buchenholz. Durch das einfache Erhitzen der Proben mit dem DSCGerät und die Auswertung der DSC-Kurven mittels multivariater Datenanalyse anstelle der herkömmlichen Enthalpie-Berechnung und univariaten Korrelation konnte der Glucosegehalt in den Proben mit einer hohen Genauigkeit und ohne jegliche Probenvorbereitung bestimmt werden. Da die DSC-Technik darüber hinaus durch die Verwendung eines Autosamplers ein sehr hohes Automatisierungspotential vorweist, stellt diese Technik eine vielversprechende Methode für die Charakterisierung der BioraffinerieRohstoffe dar.

Die vorgestellte Arbeit wurde im Rahmen des Projektes "Lokale Vorbehandlung der nachwachsenden Rohstoffe für Bioraffinerien" gefördert durch das Bundesministerium für Ernährung und Landwirtschaft (BMEL) durch die Fachagentur Nachwachsende Rohstoffe e.V. (FNR 22028411) durchgeführt.

\section{Referenzen}

[1] G. Garrote, H. Domínguez and J.C. Parajó, Hydrothermal processing of lignocellulosic materials. Holz als Roh- und Werkstoff 57: $191-$ 202, (1999); doi: 10.1007/s001070050039

[2] N. Mosier, C.E. Wyman, B.E. Dale, R. Elander, Y.Y. Lee, M. Holtzapple, Features of promising technologies for pretreatment of lignocellulosic biomass. Bioresource Technology 96 (6): 673686, (2005); doi:10.1016/j.biortech.2004.06.025 
[3] M.T. Holtzapple and A.E. Humphrey, The effect of organosolv pretreatment on the enzymatic hydrolysis of poplar. Biotechnology and Bioengineering 26: 670-676, (1984); doi: 10.1002/bit.260260706

[4] X.J. Pan, C. Arato, N. Gilkes, D. Gregg et al, Biorefining of softwoods using ethanol organosolv pulping: Preliminary evaluation of process streams for manufacture of fuel-grade ethanol and co-products. Biotechnology and Bioengineering 90: 473-481, (2005); doi: 10.1002/bit.20453

[5] P. Stockburger, An overview of near-commercial and commercial solvent-based pulping processes. Tappi Journal 76 (6): 71-74, (1993); wos: A1993LF16900010;

[6] E.K. Pye and J.H. Lora, The Alcell process - A proven alternative to kraft pulping. Tappi Journal 74: 113-118, (1991); wos: A1991EZ80700012;

[7] X. Pan, D. Xie, K.Y. Kang, S.L. Yoon and J.N. Saddler, Effect of organosolv ethanol pretreatment variables on physical characteristics of hybrid poplar substrates. Applied Biochemistry and Biotechnology, 137 (1): 367-377, (2007); doi: $10.1007 / \mathrm{s} 12010-007-9065-z$

[8] J.B. Sluiter, R.O. Ruiz, C.J. Scarlata, A.D. Sluiter, and D.W. Templeton, Compositional analysis of lignocellulosic feedstocks. 1. Review and description of methods. Journal of Agricultural and Food Chemistry 58 (16): 9043-9053, (2010); doi: $10.1021 / \mathrm{jf1} 1008023$

[9] W. Kessler, Multivariate Datenanalyse für die Pharma- Bio- und Prozessanalytik, s. 203, WileyVCH, Weinheim (2007); ISBN: 987-3-527-312627 